\title{
THIN AIRFOILS WITH SMALL TRAILING-EDGE FLAPS AT ARBITRARY ANGLES
}

\author{
E. O. TUCK ${ }^{1}$
}

(Received 2 July 1986; revised 19 August 1986 and 22 October 1986)

\begin{abstract}
The method of matched asymptotic expansions is used to solve the problem of flow over a thin airfoil possessing a trailing-edge appendage, which may be of a general character, but is confined to a region of small size compared to the airfoil's chord. A feature of this asymptotic solution is effective de-coupling of the flow problems for the main airfoil and the flap. The special case of an attached flat flap set at an arbitrary angle is solved in detail.
\end{abstract}

\section{Introduction}

Consider the linearised formula ([3], p. 171)

$$
L=-2 \rho U^{2} \int_{-l}^{l} \eta^{\prime}(x) \sqrt{\frac{l+x}{l-x}} d x
$$

for the lift per unit span of a thin airfoil of chord $2 l$ and mean surface $y=\eta(x)$, in irrotational flow of an inviscid incompressible fluid of density $\rho$, with a uniform stream $U$ at infinity. Clearly, to achieve large positive lift, we need large negative $\eta^{\prime}(x)$, especially near the trailing edge $x=+l$. Hence the conclusion, appreciated since before manned flight (and certainly before (1.1) was first derived) that highly-cambered trailing edges yield high lift. This is achieved on modern commercial wings, especially in landing configurations, by deploying trailing-edge flaps of various kinds. Such high-lift devices are discussed in standard aerodynamic reference sources such as [1], [2], [4] and [5].

\footnotetext{
'Applied Mathematics Department, University of Adelaide, G.P.O. Box 498, Adelaide, S.A. 5001, Australia.

(C) Copyright Australian Mathematical Society 1987, Serial-fee code 0334-2700/87
} 
As a simple model, consider

$$
\eta^{\prime}(x)= \begin{cases}-\alpha, & -l<x<l-h, \\ -\alpha-\beta, & l-h<x<l,\end{cases}
$$

which represents an airfoil with a flat mean surface, together with a flat attached trailing-edge flap. If $\alpha$ and $\beta$ are both small, as is necessary for validity of (1.1), the main airfoil is at angle of attack $\alpha$, and the flap is of length $h$ and is set at an angle $\beta$ to the main airfoil. The resulting lift according to (1.1) can be written

$$
L=L_{0}+L_{1} \text {, }
$$

where

$$
L_{0}=2 \pi \rho U^{2} l \alpha
$$

is the well known flat-plate lift of the main foil alone, and ([4], p. 486)

$$
L_{1}=4 \rho U^{2} l \beta\left[\arcsin \varepsilon^{1 / 2}+\sqrt{\dot{\varepsilon}(1-\varepsilon)}\right]
$$

is the additional lift due to the flap. In the above, $\varepsilon$ is the ratio between flap length and airfoil chord, i.e.

$$
\varepsilon=h /(2 l) .
$$

In most applications of interest, $\varepsilon$ is small, and in that case, (1.5) reduces to

$$
L_{1}=8 \rho U^{2} l \beta \varepsilon^{1 / 2}+O(\varepsilon) .
$$

Thus such a flap achieves a lift increase equivalent to that obtained by giving an angle of attack $\alpha$ to the whole airfoil, if the flap is set at an angle

$$
\beta=\frac{\pi}{4} \alpha / \varepsilon^{1 / 2} \text {. }
$$

Results such as (1.8) again illustrate the exaggerated influence of trailing-edge camber on lift generation. Lift increases of the order of $100 \%$ are in principle achievable by using quite small flaps at high angles of attack. There are of course both practical and theoretical limitations on this conclusion. The practical question is how to keep a real viscous fluid attached to the whole flap, and a vital consideration for design of effective high-lift devices is prevention or delay of boundary-layer separation at critical locations such as the hinge point. Such questions are beyond the scope of the present paper, and we refer to the reference sources already quoted for further discussion of the consequences for flap design.

However, even from the theoretical ideal-fluid point of view, one may suspect the validity of linearised thin airfoil theory for flap angles that are significant enough to be useful for lift augmentation. In principle, exact (i.e. non-linear or non-thin) two-dimensional airfoil theory can be used to compute the flow past an airfoil with a flap of any length, set at any angle, e.g. past the lamina $y=\eta(\dot{x})$, with $\eta(x)$ given by (1.2), and neither $\alpha$ nor $\beta$ nor $\varepsilon$ necessarily small. That is, we can solve Laplace's equation for irrotational flow past such a body, with a uniform stream and an arbitrary circulation at infinity. The appropriate circulation, and hence lift, can be determined so that the Kutta smooth-detachment 
condition can be satisfied at the flap's trailing edge, wherever it may be. However, such an analysis is very complicated and provides little insight for general airfoil configurations.

Since we are most interested in small flaps, i.e. in the limit as $\varepsilon \rightarrow 0$, even if the flap angle $\beta$ is not small, there is a compromise position between that of attempting an exact solution for the complete configuration, and that of a fully linearised theory leading to (1.1). This is provided by the method of matched asymptotic expansions [7]. In essence, we use linearised theory in an outer domain of length scale $l$, and non-linear theory in an inner domain of length scale $h$. Each of these two problems has simplified geometry, the outer geometry being that of the main airfoil and the inner that of the flap.

Indeed, to leading order, the required outer geometry can immediately be seen to be even simpler, namely (irrespective of the actual thin-airfoil geometry) that of a flat plate at zero angle of attack. That is, if $\varepsilon$ as defined above is the fundamental small parameter of this problem, any measure of the local incidence of the main foil, such as the angle of attack $\alpha$, the camber, or the thickness derivative, can be assumed to be at most of the same order as $\varepsilon$. Equivalently, any $y$-wise departure (e.g. as measured by $\eta(x)$ ) from $y=0$ can be at most of the order of $h=O(\varepsilon) l$, or else the flap would be obscured by such geometrical features. Now any linear disturbance of the order of $\varepsilon$ produces effects, on flow and hence lift, also of the order of $\varepsilon$. But we have already seen that a flap of size $\varepsilon$ produces effects of the order of $\varepsilon^{1 / 2}$ times its own angle of attack $\beta$, and we are now going to assume that the latter is not small. Hence, when solving for large effects of flaps, we can neglect the smaller (in terms of $\varepsilon$ ) effects of quantities such as the angle of attack of the main foil. More to the point, perhaps, we can delay consideration of such effects till the next $O(\varepsilon)$ term in the asymptotic expansion with respect to $\varepsilon$, at which time they are simply additive to the already-computed $O\left(\varepsilon^{1 / 2}\right)$ effects of the flap.

At first sight, the above appears to make the outer problem trivial. After all, a flat plate at zero angle of attack creates no disturbance to an inviscid fluid. Or at least it does, subject to the usual aerodynamic requirement that the flow detach smoothly from the trailing edge.

Consider, however, the flow of Figure 1. This represents a uniform stream parallel to a flat plate $y=0,-l<x<l$, about which there is non-zero circulation. The flow does not detach smoothly from the trailing edge. Instead, it is fore-and-aft symmetric, with an aft as well as a forward stagnation point, and passes around both end points with infinite velocity. This cannot happen in a real fluid, of course. But the point is, the flap tries to make it happen, and the flow shown in Figure 1 is, except for the immediate neighbourhood of the trailing edge, close to what we might hope to achieve by a suitable trailing-edge flap deployment. 


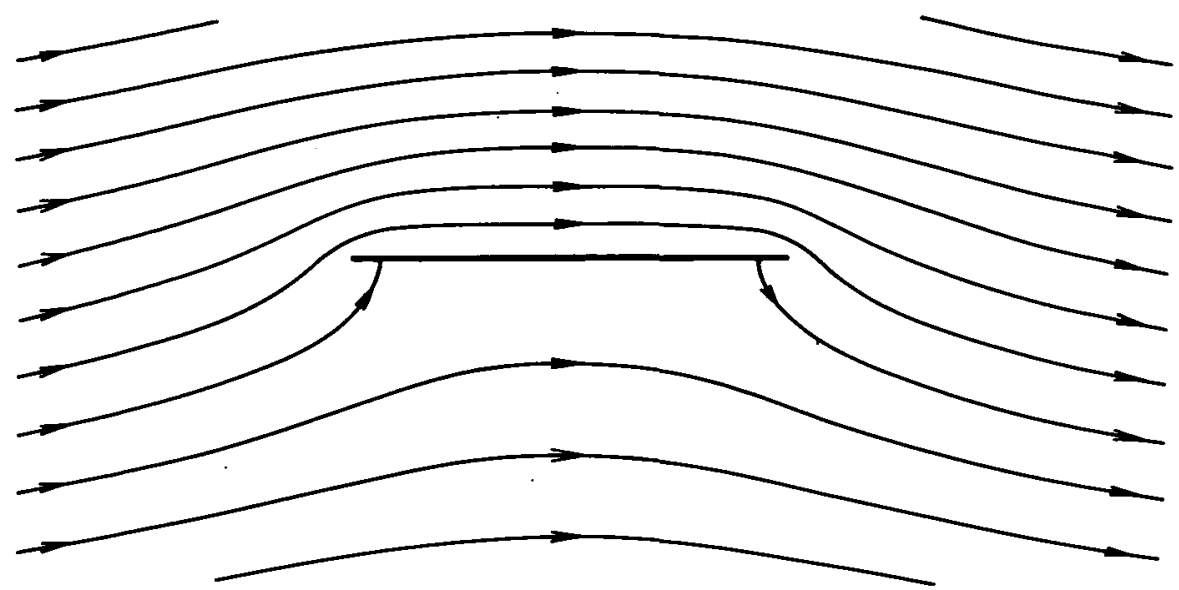

Figure 1. Streamlines for (outer) flow about a flat plate at zero angle of attack, but with non-zero circulation.

This means that, for some choice of the circulation, a flow such as that of Figure 1 solves the outer problem. All we have to do is to match with a suitable inner approximation near the trailing edge, in order to determine the circulation and hence the lift per unit span. This matching process requires us first to examine the outer flow near its trailing edge, where the outer velocity field appears to behave like the inverse square root of distance from that edge.

The inner region encompasses the complete flap, whatever may be its geometry, but loses all of the main foil except its trailing end. Thus the flap appears to be attached to the semi-infinite plate $y=0, x<l$. Clearly there is no circulation about such a semi-infinite body. Nevertheless there is a Kutta condition to be satisfied, and we can and do select from a one-parameter family of flows, a unique member that allows smooth detachment from the trailing edge of the flap.

For example, Figure 2 shows this inner solution for $\beta=\pi / 2$, i.e. for a right-angled flap. This example is used because the inner solution is simple and explicit, see equation (4.7) to follow, even though it suffers from the practical defect that a real fluid cannot negotiate the right-angled corner on the upper surface. However, more-practical flap-airfoil junctions can be treated with little increase in difficulty.

The flow of Figure 2 behaves at infinity like a uniform stream plus a flow around an edge, the latter having a velocity that decays with distance like the inverse square root. In general, a linear combination of these two flows will possess a stagnation point somewhere on the flap, and will pass around its edge with infinite velocity. However, for a suitable choice of the strength of the edge flow, the stagnation point can be moved to the edge, so yielding smooth detachment as in Figure 2. 


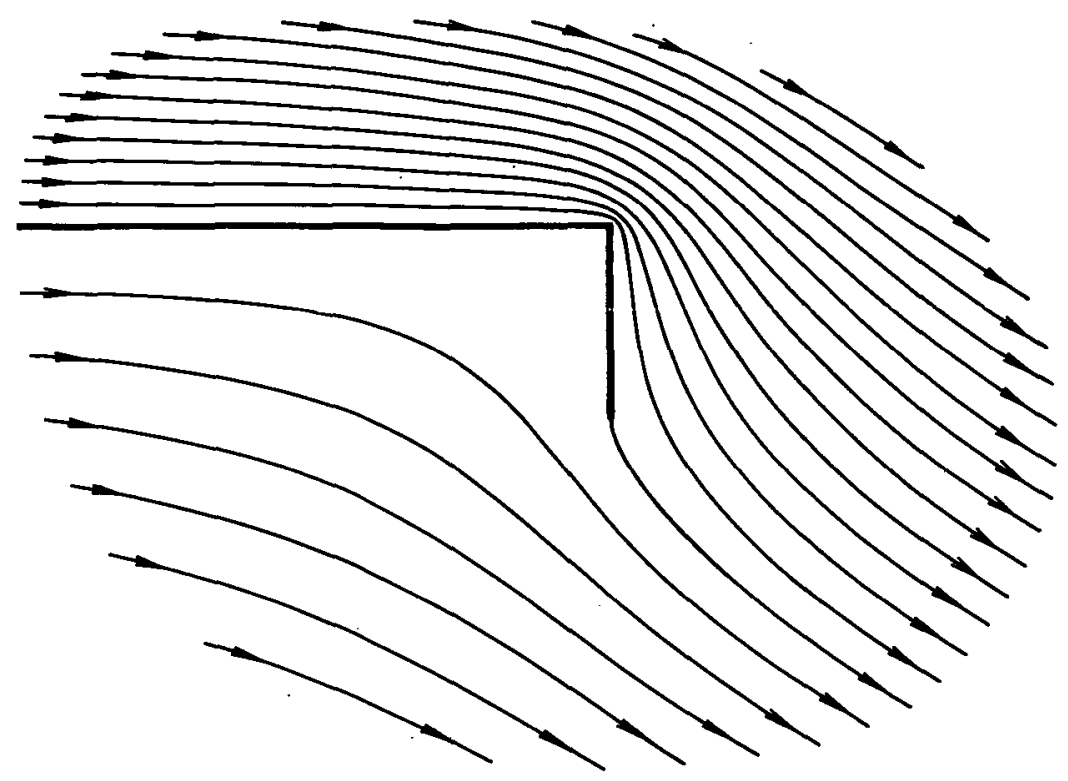

Figure 2. Streamlines for (inner) flow about a semi-infinite plate of zero thickness, with an attached flat flap at right angles.

Matching now simply asserts that the "inverse square root" velocity contribution from Figure 1 near its trailing edge is identical to that from Figure 2 near infinity. Since the latter is known, this determines the outer flow and the lift. Let us now pursue the details of these matched expansions.

\section{Formal outer expansion}

We use the complex co-ordinate $z=x+i y$ and potential $f(z)=\phi+i \psi$. The outer region is $z=O(l)$. In such a region, we assume an asymptotic expansion with respect to $\varepsilon$ of the form

$$
f(z)=U(z-l)+\varepsilon^{1 / 2} f_{1}(z)+\varepsilon f_{2}(z)+O\left(\varepsilon^{3 / 2}\right)
$$

for flow of a uniform stream $U$ over a thin airfoil whose maximum departure from the plane $y=0$ is $O(\varepsilon)$. Hence, as far as the $O\left(\varepsilon^{1 / 2}\right)$ potential $f_{1}(z)$ is concerned (but not so for $f_{2}(z)$ ), the airfoil can be replaced by the flat plate $y=0,|x|<l$. This plate can be mapped to the unit circle $|\xi|=1$ in the $\zeta$-plane by the Joukowski mapping

$$
z=\frac{1}{2} l\left(\zeta+\zeta^{-1}\right)
$$


and we suggest as our solution simply a vortex at the origin in the $\zeta$-plane, i.e.

$$
f_{1}=\frac{\kappa_{1}}{2 \pi i} \log \zeta
$$

for some choice (to be made later) of the circulation $\kappa_{1}$. The streamlines corresponding to $f=U z+\varepsilon^{1 / 2} f_{1}(z)$ with $\varepsilon^{1 / 2} \kappa_{1}=-\pi U l$ are those shown in Figure 1.

In order to proceed to the $O(\varepsilon)$ term in (2.1), we must introduce the actual shape of the airfoil surface. Suppose its upper $(+)$ and lower $(-)$ surfaces have equations

$$
y=\varepsilon \eta_{2}^{ \pm}(x)
$$

for some given functions $\eta_{2}^{ \pm}(x)$. We assume that the airfoil has a sharp trailing edge, i.e. that $\eta_{2}^{+}(+l)=\eta_{2}^{-}(+l)$ and $\eta_{2}^{+\prime}(+l)=\eta_{2}^{-1}(+l)$. Then the exact boundary condition

$$
\frac{\partial \phi}{\partial y}=\varepsilon \eta_{2}^{ \pm \prime}(x) \frac{\partial \phi}{\partial x} \quad \text { on } y=\varepsilon \eta_{2}^{ \pm}(x)
$$

linearises with $O\left(\varepsilon^{3 / 2}\right)$ error to

$$
\frac{\partial \phi}{\partial y}=U \varepsilon \eta_{2}^{ \pm \prime}(x) \text { on } y=0_{ \pm} .
$$

Hence, as we have already observed, the $O\left(\varepsilon^{1 / 2}\right)$ potential $\varepsilon^{1 / 2} f_{1}(z)$ satisfies a homogeneous boundary condition, as if the airfoil were a flat plate at zero angle of attack.

On the other hand, the $O(\varepsilon)$ potential $\varepsilon f_{2}(z)$ contains, via (2.6), contributions from $\varepsilon \eta_{2}^{ \pm \prime}(x)$. These contributions are exactly as would be obtained from linearised airfoil theory, namely

$$
f_{2}(z)=\frac{1}{2 \pi} \int_{-l}^{l}\left[m_{2}(\xi)-i \gamma_{2}(\xi)\right] \log \frac{(z-\xi)}{(l-\xi)} d \xi+\frac{\kappa_{2}}{2 \pi i} \log \zeta,
$$

where $m_{2}(x)$ is a source strength related to the thickness, i.e.

$$
m_{2}(x)=U \frac{d}{d x}\left[\eta_{2}^{+}(x)-\eta_{2}^{-}(x)\right],
$$

and $\gamma_{2}(x)$ is a vortex strength related to the camber, i.e.

$$
\gamma_{2}(x)=-\frac{U}{\pi} \sqrt{\frac{l-x}{l+x}} \int_{-l}^{l} \frac{d \xi}{x-\xi} \sqrt{\frac{l+\xi}{l-\xi}} \frac{d}{d \xi}\left[\eta_{2}^{+}(\xi)+\eta_{2}^{-}(\xi)\right] .
$$

Note that the vortex strength $\gamma_{2}(x)$ is in principle not uniquely defined, and to the expression (2.9) given above, one can add any multiple of $\left(l^{2}-x^{2}\right)^{-1 / 2}$. However, such a degree of freedom is already accounted for by the final term of (2.7), where $\kappa_{2}$ is an arbitrary constant, and $\zeta$ is as defined by (2.2). It may be 
verified directly that the expression (2.7) satisfies the boundary condition (2.6) on both top and bottom surfaces.

In effect, $f_{1}(z)$ is just the homogeneous equivalent of $f_{2}(z)$. Both $f_{1}(z)$ and $f_{2}(z)$ incorporate an arbitrary circulation about the airfoil, corresponding at infinity to an apparent line vortex. In the case of $f_{1}(z)$, this vortex is all that is responsible ifor the disturbance flow, whereas in the case of $f_{2}(z)$, there are additional contributions arising from the airfoil shape.

No Kutta condition of bounded trailing-edge velocity has been enforced at this stage. If it had been, we should have had to set $\kappa_{1}=0$, and hence $f_{1}(z) \equiv 0$. Similarly, $\kappa_{2}=0$ implies that $f_{2}(z)$ is exactly as prescribed by thin airfoil theory, since (2.9) has the property that $\gamma_{2}(+l)=0$.

For matching with the inner expansion, we shall need the behaviour of $f_{1}$ and $f_{2}$ close to $z=+l$, i.e. to $\zeta=+1$. Thus if we set $\zeta=1+\zeta_{1}$, where $\zeta_{1} \ll 1$, then

$$
z=l+\frac{1}{2} l\left(\zeta_{1}^{2}-\zeta_{1}^{3}\right)+O\left(\zeta_{1}^{4}\right) .
$$

Hence as $z \rightarrow+l$,

$$
\begin{aligned}
f_{1}(z) & =\frac{\kappa_{1}}{2 \pi i}\left(\zeta_{1}-\frac{1}{2} \zeta_{1}^{2}\right)+O\left(\zeta_{1}^{3}\right) \\
& =\frac{\kappa_{1}}{2 \pi i} \sqrt{\frac{z-l}{l / 2}}+O(z-l)^{3 / 2} .
\end{aligned}
$$

A similar but not so immediate conclusion, with a different error term, applies to $f_{2}(z)$, namely as $z \rightarrow+l$,

$$
f_{2}(z)=\frac{\kappa_{2}}{2 \pi i} \sqrt{\frac{z-l}{l / 2}}+O(z-l) .
$$

The non-zero contribution to (2.12) comes, as with (2.11), from the last term of (2.7). The integral in (2.7) has been designed so that it contributes nothing as $z \rightarrow+l$. In the first place, the factor $(l-\xi)$ in the denominator of the logarithm simply contributes a physically-irrelevant constant, such that, providing the integral converges at $z=+l$, its value is zero. Convergence of the integral for $f_{2}(z)$ itself is not really an issue, a more severe test being a demand that the corresponding integral for the derivative $f_{2}^{\prime}(z)$ converges as $z \rightarrow+l$. If this is so, then the error term in (2.12) is as written. Convergence of the integral for $f_{2}^{\prime}(z)$ requires that $m_{2}(\xi)-i \gamma_{2}(\xi)$ tends to zero as $\xi \rightarrow+l$. This is true for $\gamma_{2}(\xi)$ by construction, noting that of all possible members of the one-parameter family of vortex strengths, only the particular solution (2.9) has this property. The requirement of a sharp trailing edge implies that $m_{2}(+l)=0$, and this condition is necessary for validity of (2.12). 
In summary, combining (2.1), (2.11) and (2.12) with $z-l=O(\varepsilon l)$, the inner expansion of the outer expansion is of the form

$$
f(z)=U(z-l)+\frac{\varepsilon^{1 / 2} \kappa_{1}}{2 \pi i} \sqrt{\frac{z-l}{l / 2}}+\frac{\varepsilon \kappa_{2}}{2 \pi i} \sqrt{\frac{z-l}{l / 2}}+O\left(\varepsilon^{2}\right) .
$$

The first two terms of (2.13) are $O(\varepsilon)$, and the third is $O\left(\varepsilon^{3 / 2}\right)$.

The constants $\kappa_{1}$ and and $\kappa_{2}$ will now be determined by matching with the inner flow near the trailing edge. Once this is done, any outer flow quantity required is available with error $O\left(\varepsilon^{3 / 2}\right)$. In particular, the net circulation about the airfoil is

$$
\kappa=\varepsilon^{1 / 2} \kappa_{1}+\varepsilon\left[\kappa_{2}+\int_{-l}^{l} \gamma_{2}(x) d x\right]+O\left(\varepsilon^{3 / 2}\right),
$$

from which the lift per unit span,

$$
L=-\rho U \kappa,
$$

follows by the Kutta-Joukowski theorem. Note that if $\kappa_{1}=\kappa_{2}=0$, then $L$ is given by (1.1), with the camber function

$$
\eta(x)=\frac{1}{2} \varepsilon\left[\eta_{2}^{+}(x)+\eta_{2}^{-}(x)\right] .
$$

\section{Formal inner expansion}

The inner region is defined by $z-l=O(h)=O(\varepsilon l)$. Hence we may define an inner coordinate $Z$ by

$$
z=l+h Z \text {. }
$$

The potential is assumed to have an asymptotic expansion in $\varepsilon$ of the form

$$
f(z)=2 l U\left[\varepsilon F_{2}(Z)+\varepsilon^{3 / 2} F_{3}(Z)+O\left(\varepsilon^{2}\right)\right] .
$$

Note that the fact that (3.2) starts with a term of $O(\varepsilon)$ simply corresponds to the $O(\varepsilon l)$ length scale of the inner flow; however, it also can be anticipated from a need to match (2.13).

Specifically, matching with (2.13) demands that as $Z \rightarrow \infty$,

$$
F_{2}(Z) \rightarrow Z+i K_{2} Z^{1 / 2}
$$

and

$$
F_{3}(Z) \rightarrow i K_{3} Z^{1 / 2}
$$

where

$$
K_{2}=-\frac{\kappa_{1}}{2 \pi U l}
$$


and

$$
K_{3}=-\frac{\kappa_{2}}{2 \pi U l} \text {. }
$$

That is, the flow corresponding to $F_{2}(Z)$ appears at infinity like a unit uniform stream combined with a " $Z^{1 / 2}$ " potential, which is the exact solution for flow around a semi-infinite plate $Y=0_{ \pm}, X<0$. A similar description, but without a uniform stream component, applies to $F_{3}(Z)$. The apparent semi-infinite plate at infinity is the inner representation of the trailing edge of the main airfoil.

To this plate is attached a trailing-edge flap configuration of arbitrary geometry. That is, the inner task is to solve for flow around the end of a semi-infinite plate, to which is appended a body of, in principle, any shape whatever. Note that we have required here that the upstream semi-infinite plate be of zero thickness. The somewhat more general problem where the thickness of the main airfoil does not vanish at its trailing edge would lead to an inner problem with a finite-thickness plate upstream, and is left for future investigation.

In the present case, the whole inner flow is forced by the upstream conditions (3.3), (3.4) above, and we can write

$$
F_{2}(Z)=F(Z)+K_{2} G(Z)
$$

and

$$
F_{3}(Z)=K_{3} G(Z)
$$

where, as $Z \rightarrow \infty$,

$$
F(Z) \rightarrow Z
$$

and

$$
G(Z) \rightarrow i Z^{1 / 2}
$$

Both $F(Z)$ and $G(Z)$ can be determined uniquely for any specified appendage geometry.

At this stage, the constants $K_{2}$ and $K_{3}$ are not determined, and hence each solution $F_{2}(Z)$ and $F_{3}(Z)$ is obtained as a one-parameter family. It is time to introduce a Kutta condition. Suppose first that the appendage itself possesses one sharp trailing edge, as would be the case for a single attached flap, located at $Z=Z_{0}$. Then, in general, the potentials $F(Z)$ and $G(Z)$ will each demand infinite velocity at $Z=Z_{0}$. Since $F_{3}(z)$ is directly proportional to $G(Z)$, this singularity is removable for $F_{3}(Z)$ only by removing the flow altogether, i.e. by setting $K_{3}=0$.

However, for $F_{2}(Z)$, one must expect that there exists a non-zero unique value of $K_{2}$ such that $\left|F_{2}^{\prime}\left(Z_{0}\right)\right|<\infty$. Physically, as is usual in airfoil theory, the flow $F_{2}(Z)$ contains a stagnation point whose location $Z=Z_{S}\left(K_{2}\right)$ depends on the parameter $K_{2}$. By a suitable choice of $K_{2}$, we can force $Z_{S}=Z_{0}$, the resulting flow having neither zero nor infinite velocity at that point. 
If there is more than one sharp trailing edge associated with the appendage configuration, it can be assumed that this configuration is multiply connected, consisting of one or more detached flaps, the number of trailing edges being one more than the number of flaps, i.e. one trailing edge for each flap, plus one for the main airfoil. Any other situation is indeterminate, in un-separated irrotational flow. Thus each flap now has in effect its own circulation and its own Kutta condition, but there is one Kutta condition left over, and this determines $K_{2}$ uniquely.

In summary, whatever may be the appendage configuration (subject to the above restriction, if non-simply connected), the inner flow will determine $K_{2}$ uniquely, and demand $K_{3}=0$. This means that, returning to the outer domain, the lift is the sum of an $O\left(\varepsilon^{1 / 2}\right)$ contribution proportional to $\kappa_{1}=-2 \pi U l K_{2}$ and an $O(\varepsilon)$ contribution, which (since $\kappa_{2}=0$ ) is precisely that for the main airfoil with no flap, i.e. is given by (1.1).

The remaining task is thus to find a flow $F_{2}(Z)$ over the exact flap surface, satisfying (3.3) at infinity, and appropriate Kutta conditions. Although this is still a major computational task, it is often considerably simplified relative to the full non-linear problem, in which the airfoil geometry also enters. Indeed, even aside from the computational simplification, the de-coupling of airfoil and flap geometries achieved by the present analysis is of design significance.

\section{The simple attached flap}

There are some cases in which the inner flow can be solved analytically, or in which at most a quadrature is required, and the simple attached flap of Figure 3 is one important example. The flow domain in the complex potential plane has a cut along the negative real axis, and hence can be mapped to the upper half $t$-plane by

$$
F_{2}=-k t^{2}
$$

for some some real positive $k$. Schwartz-Christoffel considerations then suggest that the solution is given by integration of

$$
\frac{d Z}{d t}=-2 k t\left(\frac{t+1}{t-\lambda}\right)^{\beta / \pi},
$$

where $\lambda$ is another real positive constant. 

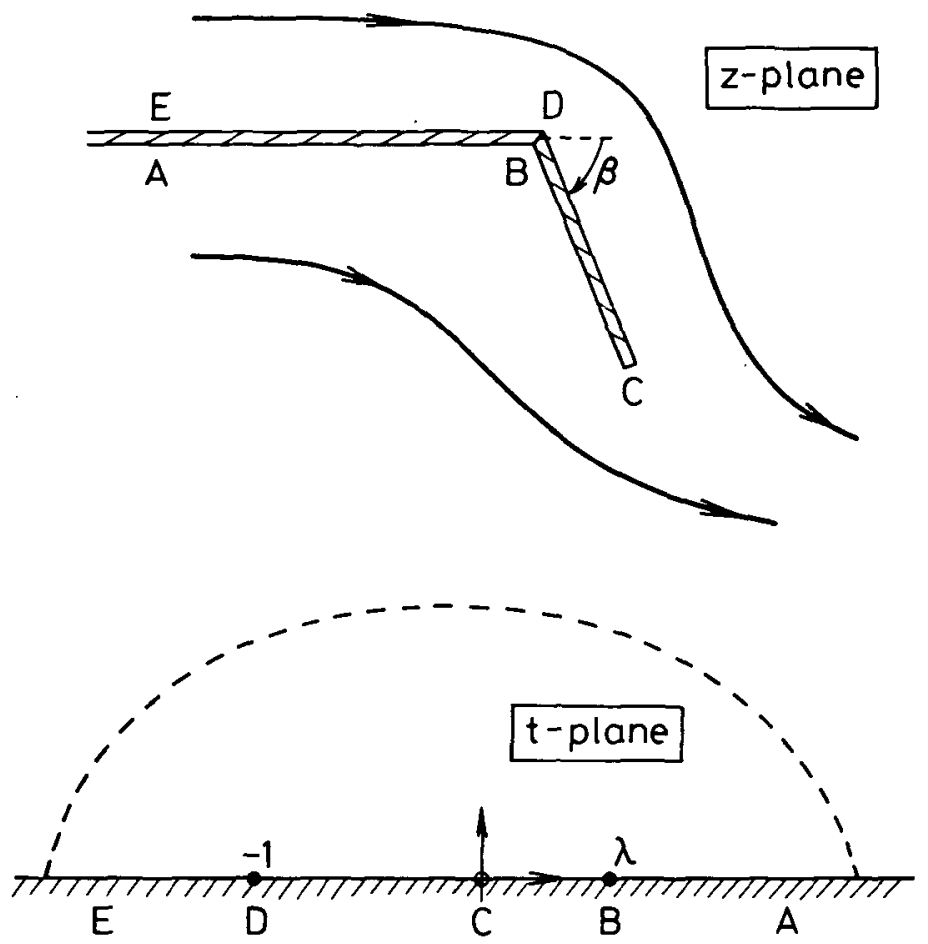

Figure 3. Flow domain in the $z$ and $t$ planes for an attached flap at angle $\beta$.

The upper $(D)$ and lower $(B)$ corners map to $t=-1$ and $t=\lambda$ respectively, and, since the complex velocity is

$$
\frac{d F_{2}}{d Z}=\left(\frac{t-\lambda}{t+1}\right)^{\beta / \pi}
$$

the correct change of $\mp \beta$ radians in the flow direction is achieved at both of these points. These points $D$ and $B$ have (respectively) infinite and zero velocity, if $\beta>0$. In contrast, the end point $C$ with $t=0$ has finite velocity, so that the Kutta condition is satisfied. The far-field boundary condition (3.3) is satisfied as $t \rightarrow \infty$, with

$$
K_{2}=\frac{2 \beta}{\pi}(1+\lambda) \sqrt{k} .
$$

All of the above applies for any $k, \lambda>0$, and we need two more equations in order to determine these two parameters. In fact, for a general choice of $k$ and $\lambda$, the solution (4.2) corresponds to a more general flap configuration than that of Figure 3 since so far we have not ensured that the upper and lower corner points $D$ and $B$ be at the same point of space in the physical plane. This is now achieved 
simply by demanding that the distance $C D$ between edge and upper corner equals that $C B$ between edge and bottom corner. Setting both of these distances equal to unity (as we are entitled to do, since the actual flap length $h$ has been scaled out by (3.1)) gives us the required two equations, namely (after division by $2 k$ )

$$
\begin{aligned}
-\int_{-1}^{0} t\left(\frac{1+t}{\lambda-t}\right)^{\beta / \pi} d t & =\int_{0}^{\lambda} t\left(\frac{1+t}{\lambda-t}\right)^{\beta / \pi} d t \\
& =\frac{1}{2 k} .
\end{aligned}
$$

Equation (4.5) is a single transcendental equation to determine $\lambda$, after which (4.6) determines $k$.

For a general flap angle $\beta$, the integrals in (4.5) must be evaluated by numerical quadrature, but for $\beta=\pi / 2$, i.e., a right-angled flap, they can be evaluated in closed form, and we find $\lambda=\frac{1}{3}$ and $k=\sqrt{3}$. Indeed, the whole solution then is in closed form, namely

$$
Z=-(t+1)^{3 / 2}(3 t-1)^{1 / 2}
$$

Streamlines $\mathscr{F} F_{2}=$ constant computed by combining (4.1) (with $k=\sqrt{3}$ ) and (4.7) are those given in Figure 2.

For $\beta \neq \pi / 2$, having evaluated both integrals in (4.5) numerically with $\lambda$ as input, we choose $\lambda$ so that they equal each other. A plot of $\lambda$ and $k$ versus $\beta$ is given in Figure 4. Also shown is the ratio

$$
\frac{L}{L_{1}}=\frac{\pi}{4} K_{2} / \beta=\frac{1}{2}(1+\lambda) \sqrt{k}
$$

between the $O\left(\varepsilon^{1 / 2}\right)$ lift $L$ computed from (2.14) and (2.15) according to the present non-linear theory, and the value $L_{1}$ given by the linearised theory, as in (1.7). Note that this comparison of the present finite- $\beta$ lift is with a fully linearised aproximation, where the mean surface is given by (1.2) and the lift by (1.7). No comparison is made with a "pseudo-nonlinear" result that could be obtained by replacing $\alpha, \beta$ and $h$ in (1.2) by $\tan \alpha, \tan \beta$ and $h \cos \beta$ respectively, without amendment of the aerodynamics. Since $L_{1}$ is just the limit of $L$ as $\beta \rightarrow 0$, the ratio $L / L_{1}$ starts at unity at $\beta=0$, and it decreases with $\beta$. That is, the linearised theory over-predicts the lift, but not by very much. For example, its error is less than $3 \%$ up to $\beta=45^{\circ}$, and only $12 \%$ at $\beta=90^{\circ}$, well beyond the practical range of validity for attached flow.

Figure 4 includes results for "folded-back" flaps with $\beta>90^{\circ}$, even though these are only of theoretical interest. For example, we find that $L / L_{1} \rightarrow \frac{1}{2}$ as $\beta \rightarrow 180^{\circ}$, i.e. when the flap is folded right back flat underneath the main foil. 


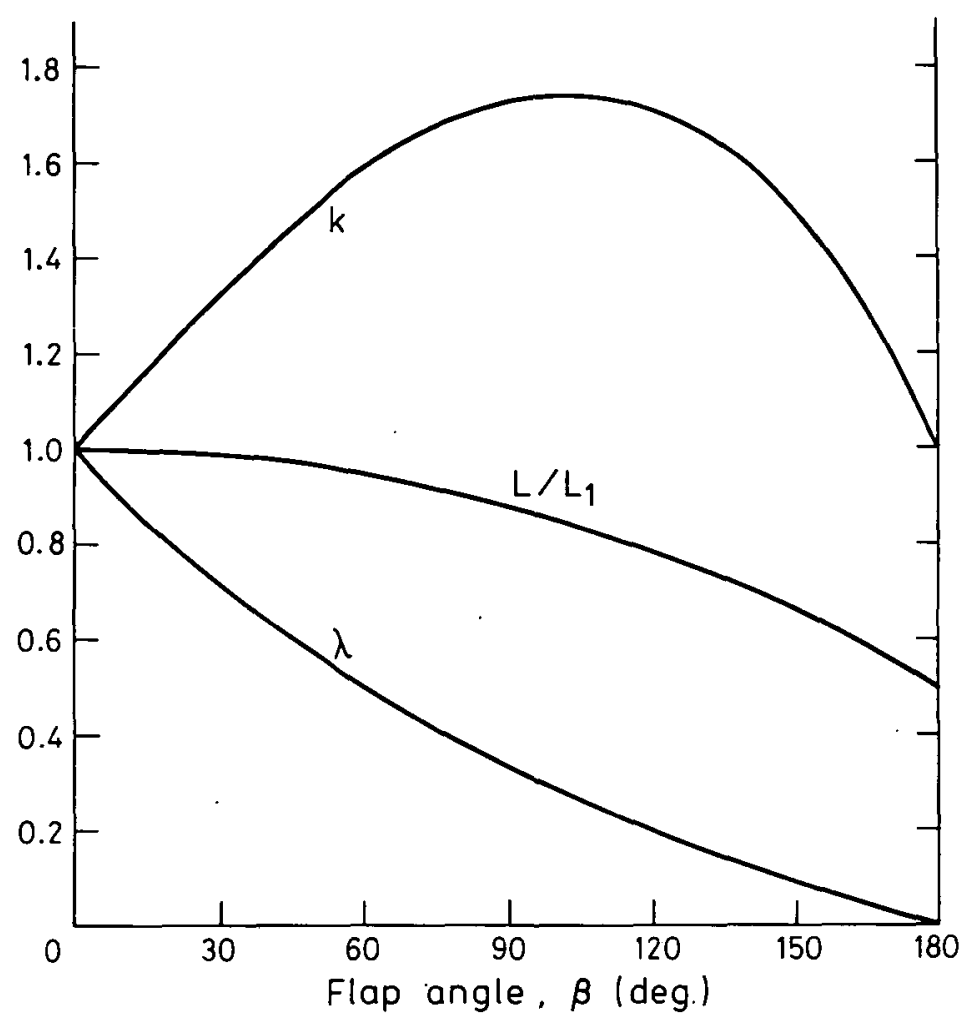

Figure 4. Variation with flap angle $\beta$ of the parameters $k$ and $\lambda$ of the inner mapping for a flat attached flap, and of the lift reduction factor $L / L_{1}$ relative to that given by linearised theory.

Note that this situation still in theory yields non-zero lift, since the Kutta condition has been enforced at the location of the flap end, not at the airfoil end, and hence a non-zero circulation is created. However, the lift at $\beta=180^{\circ}$ is somewhat reduced relative to that for a deployed flap. Maximum lift (of about $\left.14 \rho U^{2} l \varepsilon^{1 / 2}\right)$ is achieved at $\beta \approx 149^{\circ}$; i.e., this is the flap angle that turns an inviscid fluid most effectively around the trailing edge of the main foil. Although such a flow could not be achieved by a flat attached flap in a real viscous fluid, it is instructive in setting bounds on achievable lift for flap configurations that do permit attached flow.

\section{Conclusion}

The purpose of the present paper has been to outline a systematic matched asymptotic expansion approach to study of trailing-edge appendages of small size but at arbitrary incidence. Only the case of a simple flat attached flap has been 
treated in detail, the results agreeing with linearised theory in the limit of small flap angle.

For general flap configurations, a significant conclusion is that the flows over the main airfoil and the flap can be treated separately, and then matched together. Both problems in general demand numerical solution, but this asymptotic procedure provides a valuable de-coupling of the geometrical complexities in these two differently-scaled problems. For example, we should expect that details of the main airfoil's leading-edge geometry would influence the performance of the trailing-edge flap in only a minor way, and the present theory builds in such a property, by replacing the main airfoil with a semi-infinite plate, when analysing the inner flow about the flap.

There are many possible extensions of this work. Detached and multiple flaps are easy to treat within this same non-linear framework. This would generalise existing linearised theories (e.g. [8], [9]) for detached flaps or tandem airfoils. The work also has relevance to models of bluff-body aerodynamics and (especially) hydrodynamics, in which one assumes separated flows, the main body being thin and allowing a linearised treatment, but the trailing end having a transom or wedge shape from which a nonlinear constant-pressure wake or cavity is shed. Three-dimensional applications, such as to wing tips with winglets, are also under investigation in the context of yacht keels. Ground effect aerodynamics [6] provides another interesting extension in which, when the ground clearance is small, even the outer problem is nonlinear.

\section{References}

[1] I. H. Abbott and A. E. von Doenhoff, Theory of Wing Sections (Dover, New York, 1959).

[2] D. M. McRae, “Aerodynamics of mechanical high-lift devices", in Assessment of Lift Augmentation Devices (ed. J. William \& P. E. Colin) NATO AGARD Lecture Series 43 (1970).

[3] J. N. Newman, Marine Hydrodynamics (MIT Press, Cambridge Mass., 1977).

[4] H. Schlichting and E. Truckenbrodt, Aerodynamics of the Airplane (McGraw-Hill, New York, 1979).

[5] B. Thwaites, Incompressible Aerodynamics (Oxford University Press, Oxford, 1960).

[6] E. O. Tuck, "Steady flow and static stability of airfoils in extreme ground effect", J. Engrg. Math. 15 (1981) 89-102.

[7] M. Van Dyke, Perturbation Methods in Fluid Mechanics (Academic Press, New York, 1964).

[8] G. D. Watt and G. V. Parkinson, "On the application of linearized theory to multi-element aerofoils. Part 1. Tandem flat plate aerofoils", Aero. Quart. 34 (1983), 46-60.

[9] R. B. White and M. T. Landahl, "Effect of gaps on the loading distribution of planar lifting surfaces,” AIAA J. 6 (1968) 626-631. 